\title{
Blockchain for the Future Development of the Pharmaceutical Industry
}

\author{
Renfei Gao ${ }^{1, *, \dagger}$, Xiao Yu ${ }^{2, \dagger}$, Zhirui Zhang ${ }^{3, \dagger}$ \\ ${ }^{1}$ Economics, School of Economics and Management, Southwest University, Chongqing, China \\ ${ }^{2}$ Mathematics and Applied Mathematics, School of Mathematics, Sichuan University, Chengdu, Sichuan, China \\ ${ }^{3}$ Business Economics, School of Social Sciences, University of California Irvine, California, U.S. \\ *Corresponding author. Email: g1586177672@email.swu.edu.cn \\ These authors contributed equally.
}

\begin{abstract}
Blockchain is an emerging industry, and its applications are well established in product traceability, data circulation, supply chain management, judicial depository, government data sharing, and livelihood services. In China, blockchain technology has great value in the medical industry and influences its future development trend in the medical industry. This study explores the impact of blockchain on the healthcare industry and how consumer attitudes toward using blockchain affect its future development. This paper further develops the impact of blockchain on the pharmaceutical industry, using COVID-19 as an example to illustrate the highly developed and advanced application of blockchain technology in the healthcare industry. Validated factor analysis and Statistical Product and Service Solutions structural equation modeling was performed using by Wen Juan Xin. This study concludes that the recognition and acceptance of blockchain technology applications in the healthcare industry are clarified and reveal various problems in the current applications. Therefore, decision-makers in healthcare industry organizations or sectors need to carry out reforms related to blockchain applications, especially privacy protection and information security enhancement, to provide reference and higher and more stringent requirements among blockchain applications.
\end{abstract}

Keywords: Emerging Industry, Healthcare, COVID-19, Blockchain, Pharmaceutical Industry

\section{INTRODUCTION}

With the progress and innovation of science and technology, the application of blockchain has gradually penetrated many fields of economy and society. It has been well applied in product traceability, data circulation, supply chain management, judicial depositing, government data sharing, people's livelihood services, and other fields. Blockchain plays an obvious role in promoting economic and social development.

Blockchain is an important part of the next generation of information technology. In 2008, an academic (team) under the pseudonym "Satoshi Nakamoto" first proposed a digital currency called Bitcoin in the Cryptography mailing list. In other words, without a central authority organization, people who do not trust each other can use Bitcoin to conduct transactions. Blockchain is derived from the underlying technology of Bitcoin. Blockchain technology (Blockchain 1.0), the core technology of the Bitcoin trading system, has been widely used.

Blockchain can be termed meta-technology because it results from the integration of several other technologies such as software development, cryptographic technology, and database technology [1]. Blockchain technology is characterized by decentralization, high trust, and distributed technical solutions.

At present, more and more scientific research institutions and technology companies at home and abroad participate in the research of blockchain and comprehensively apply blockchain technology in technological change and industrial upgrading. The initial application of blockchain technology is the original public ledger of bitcoin, which has later inspired other implementations called altchains. These kinds of networks also provide trust-based services that are not limited to currency transactions [2].

The application and development of blockchain technology play an important role in promoting the 
development of the new generation of the information technology industry. In recent years, in the context of China's "'13th Five-Year' National Informatization Plan ", "'13th Five-Year' National Population Health Informatization Development Plan, "and other favorable policies, blockchain technology is becoming the focus of national information construction. As a new field of blockchain application, the medical industry has received high attention. However, there are some inaccurate concepts, evaluations, and expectations regarding the application of blockchain technology. The potential value of the technology in the medical industry may cause some misunderstanding. These problems are worthy of public attention so that this paper can correctly use blockchain technology and look into the development trend of China's future medical industry.

In December 2019, some medical institutions in Wuhan, Hubei province, reported patients with pneumonia of unknown cause. Novel Coronavirus 2019 pneumonia was classified as a Class B infectious disease in China under the control of Class A infectious disease [3]. However, there are a corresponding number of confirmed cases every day, indicating the necessity and urgency of standardized diagnosis and treatment. China's COVID-19 diagnosis and treatment program relies on mobile Internet technology and blockchain technology [4]. Through the blockchain system, early screening of COVID-19, effective and reasonable allocation of medical resources, active implementation of emergency medical rescue and joint diagnosis and treatment by medical confederate Internet remote experts, etc. It provides some reference for the prevention and control of novel coronavirus, a major health event in China.

Nowadays, the application and development of health and big medical data involve many fields, such as clinical diagnosis and treatment, medical, scientific research, public health, chronic disease management, and artificial medical intelligence. The healthcare industry faces challenges in data security, privacy protection, data sharing, and interoperability.

The distributed storage, tamper-free, encrypted storage, and other features of blockchain technology bring technical breakthroughs to break the bottleneck problems such as data isolated island and medical data sharing. It also provides appropriate solutions to the pain points and difficulties of data application in health and medical care. At the same time, it provides basic technical support for improving the level of medical service.

In recent years, especially in the severe period of COVID-19, the application of blockchain technology in the medical industry has been highly developed and improved. The rational allocation of medical resources based on blockchain technology, expert consultation through the Internet Medical Alliance, electronic medical records, big data travel code, and the application of health code have all brought great convenience to the medical industry. At the same time, further use of the technology has raised concerns about data security, privacy, data sharing, and operability. The overall application of blockchain technology in the medical industry has been investigated and studied, and the overall conclusion is that the convenience brought by this application is significantly greater than some of the hidden dangers. However, the existing research results often ignore the opinions and views of the major participants and beneficiaries of the medical industry -the consumers (or the recipients of medical services) of the medical market. Therefore, our research mainly focuses on the perspective of medical service receivers to investigate and analyze whether the receivers support the increasingly in-depth application of blockchain technology in the medical industry today.

The COVID-19 epidemic has posed a great threat to people's safety. Under this background, people began to pay more and more attention to the medical industry. Blockchain technology, as an emerging technical field, its application can provide the medical industry with faster and more convenient than ever before so that people can better get the guarantee of medical services and get greater benefits. Therefore, it is reasonable to expect that health care service recipients as a whole will support blockchain technology because they benefit from the convenience of its application but will have concerns due to issues such as transparency and privacy security.

To confirm our point of view and try to find some innovative suggestions on blockchain application in the medical industry, this paper chose a questionnaire to conduct the research. The questionnaire mainly involved questions about the understanding and support of the technology, concerns about privacy leakage and information transparency, and suggestions on the application of the technology in the medical field Through the collection and sorting of questionnaire data, it can be concluded that the attitude of medical service receivers towards the application of blockchain technology in the medical industry is basically consistent with the hypothesis, and they put forward some suggestions in the questionnaire.

\section{LITERATURE REVIEW}

In recent years there has been an increasing amount of literature on the blockchain because blockchain belongs to the category of new infrastructure in the world. It belongs to the new infrastructure information infrastructure because blockchain is a new way of asymmetric encryption algorithm, consensus mechanism, distributed storage, peer-to-peer transmission, and other related technologies. Therefore, blockchain can be 
applied to many fields with broad applications and has good development prospects [5].

\subsection{Key Definition}

There are many technical features of blockchain, so blockchain is getting more and more attention. However, the research will not show all the lists. Instead, it will explain those features that mainly benefit the pharmaceutical industry. [LB1] The first is invariance. It cannot be edited, deleted, or updated by any user on the network. Secondly, decentralization means storing anything starting from cryptocurrencies, important documents, contracts, or other valuable digital assets. Because a blockchain doesn't require any administrative privileges, blockchain would access it and store the assets directly from the web. Third, with enhanced security, blockchain provides stronger protection for users. It provides a special kind of camouflage cryptography, as cryptography in region refinement provides another layer of protection for users. That brings more security to the user's data. The blockchain industry includes upstream hardware, technology, and infrastructure; midstream blockchain application and technology services; and downstream blockchain application areas [6]. This paper will be conducting an in-depth study of the pharmaceutical industry to understand the pros and cons of blockchain for the pharmaceutical industry and consumer perceptions of blockchain use in the pharmaceutical industry. The pharmaceutical industry plays a vital role in every country, including the pharmaceutical industry and patient privacy and security [7].

\subsection{Implication}

Because of the unique nature of the pharmaceutical industry, hospitals or private clinics have access to a large amount of confidential information about patients and their current physical conditions. The privacy, security, and tamper-proof nature of blockchain can protect consumers' personal information. Because blockchain differs from a typical database, it stores information; blockchain stores data in blocks and links them together. As new data comes in, it is fed into a new block. Once the block is filled with data, it is linked to the previous block, making the data linked together in chronological order. And decentralized blockchains are immutable, which means that the input data is irreversible. In modern areas, digital information flows from one end to the other through untrusted transmission channels. Here, privacy and confidentiality are major concerns. Blockchain technology provides secure peer-to-peer communication. In blockchain technology, transactions are publicly available, but they cannot be modified once recorded. For the consumer's uses the uniformly designed questionnaire to collect information from the respondents, ask for their opinions, collect the information, sort out the analysis, and physical condition, the transaction is permanently recorded and cannot be tampered with. In addition, $\mathrm{He}$ and Wang, in the article "Analysis of informatization application of medical management base on blockchain technology," carried out in favor of explaining that medical management informatization is an essential means to improve the health of all people under the special national conditions of a large population and scarce medical resources in China. And blockchain technology solves the problems of interconnection and interoperability of medical information resources, information confidentiality. It cannot tamper with ability, and information sharing, which is an essential technical support for the further development of the Internet and medical care in China. Consumers will have more peace of mind when using the Internet because blockchain technology will protect their privacy. Pharmaceutical companies can also use the confidentiality technology of blockchain to gain protection for their interests. However, there are still objections, and these can be negative for consumers. In the article "Security Problems on Blockchain: The State of the Art and Future Trends," Han and others took intensive research at blockchain security [8]. They argued in favor of the viability of blockchain but also list security problems with it. First, code vulnerability they used Bitcoin to explain that blockchains are vulnerable. Some cryptographic components can also be compiled with flaws and vulnerabilities that cause the trading platform to believe that the original transaction was not validated by the miners and generate a new transaction to pay the attacker again. If the attack is successful, the attacker gets double the bitcoins. The second privacy protection problem is that the data layer privacy protection technology provides basic privacy protection for users and transactions in the blockchain from the data structure perspective. Still, it cannot avoid the correlation between trades and user IP addresses in network transmission, leading to the attacker using listening and tracking IP addresses to infer the relationship between transactions and public key addresses, undermining the blockchain. This undermines the privacy protection goal of blockchain. These same issues can also affect the use of blockchain by pharmaceutical companies and or consumers in the event of an accident [9].

\section{METHOD}

In this paper, the method of questionnaire survey was used to collect and analyze data. Because the survey sample range is large and the quantity is large. It helps eliminate the random error, which is difficult to avoid by means of measurement, and makes the research results from universal, objective, and persuasive. A questionnaire survey is a survey method. The designer

conclude. It is a tool for collecting data in social investigation and research. 
This paper takes the Chinese medical market as the object, investigates the relevant situation in the current medical market, the understanding of the blockchain technology, and analyzes the current status and problems of the application of the blockchain in the medical market, as well as the views of the entire medical industry environment on the application of the blockchain technology in the development. To test the hypotheses empirically, an online questionnaire was sent via a mobile phone app called Wen Juan Xing to approximately 300 meat consumers, and 192 participated in the survey. After sample screening, data consolidation, and logical test, and eliminating the questionnaires with logical errors or inconformity with the research requirements, the final remaining effective sample size was 151 . The questionnaire was distributed and collected through the online mobile APP, which applied convenience sampling. To obtain and establish a representative sample, this research intentionally sent it out to people of various age groups and regions.

Firstly, the reliability and validity of measurements are analyzed in this paper. Reliability test, also known as reliability test, is the method of repeated measurement to repeatedly verify the measurement results and measure the consistency degree. In this paper, SPSS was used to test the A reliability coefficient. Specifically, the reliability test method of Cronbach $\alpha$ coefficient is adopted.

The Cronbach $\alpha$ reliability coefficient of the questionnaire was 0.833 , indicating that all the above variables had good reliability and consistent stability.

Secondly, difference analysis is carried out on the measurement results. According to the analysis of age variance, some loopholes in China's current medical industry system under different age conditions. At present, the optimization of the medical industry system cannot fundamentally change the status quo. Respondents have a certain understanding of blockchain technology, and blockchain technology can significantly improve the credit of the medical industry system. The P-value of which link of the medical industry system the blockchain technology is most helpful for credit improvement is far greater than 0.05 , indicating no significant difference in these issues among different age groups.

In this paper, multiple regression analysis was performed on the measured results. The survey mainly investigated the application of blockchain technology in the medical industry in China from six aspects: privacy, security, tamper-proof, convenience, accuracy, data release, and storage of medical record data.

The questionnaire used in this study was in the form of a five-level scale, and five answer options were set for the subjects to make the only choice. The five answer choices 1, 2, 3, 4, and 5 represent the degree from dissatisfied to satisfied. When creating the original SPSS data file, the questionnaire code should set the corresponding variable value label according to this.

Table 1. The reliability analysis results of the total table

\begin{tabular}{ccc}
\hline N of Items & $n$ & Cronbach $\alpha$ \\
\hline 12 & 151 & 0.833 \\
\hline
\end{tabular}

Table 2. ANOVA (Simplified format)

\begin{tabular}{cccccc}
\hline Age $($ Mean \pm Std. & & & & & \\
& Tamper-proof & Convenience & Good storage & Accuracy \\
Deviation) & & Security & sex & & \\
\hline$F$ & 0.272 & 0.590 & 0.580 & 0.093 & 0.208 \\
$p$ & 0.763 & 0.558 & 0.564 & 0.911 & 0.813 \\
\hline
\end{tabular}

Table 3. Descriptive Analysis

\begin{tabular}{cccccc}
\hline Items & N of samples & Min & Max & Mean & Std. Deviation \\
\hline Blockchain awareness & 151 & 1 & 5 & 2.680 & 1.039 \\
Blockchain acceptance & 151 & 1 & 5 & 3.420 & 0.785
\end{tabular}


Table 3. Descriptive Analysis

\begin{tabular}{ccccccc}
\hline Items & N of samples & Min & Max & Mean & Std. Deviation & Median \\
\hline $\begin{array}{c}\text { Understanding of the application of } \\
\text { blockchain in the healthcare market }\end{array}$ & 151 & 1 & 5 & 2.620 & 1.159 & 3 \\
Privacy & 151 & 1 & 5 & 2.740 & 1.157 & 3 \\
Security & 151 & 1 & 5 & 2.380 & 1.088 & 3 \\
Tamper-proof sex & 151 & 1 & 5 & 2.920 & 0.966 & 4 \\
Convenience & 151 & 1 & 5 & 3.780 & 0.901 & 3 \\
Good storage & 151 & 1 & 5 & 3.300 & 0.953 & 4 \\
Accuracy & 151 & 1 & 5 & 3.820 & 0.910 & 4
\end{tabular}

\section{RESULT}

Through the questionnaire survey on consumers' understanding, support, information security, privacy protection, and other aspects of blockchain technology, we could understand the health care service recipients' perception of the application of blockchain technology in the healthcare industry. The paper first summarizes the results of the questionnaire survey. First of all, although not everyone has some understanding of blockchain technology, they have more or less a general understanding of the application of this technology in the medical industry under the influence of the current COVID-19. At the same time, the convenience and accuracy brought by the application of this technology in the medical industry have also been more consistent recognition. However, The main point of disagreement is the privacy and security of the technology in use. Overall, more people choose to believe that the technology can ensure adequate privacy and security (especially in light of the current COVID-19 epidemic). This perception also correlates with age, with surveys showing that older groups tend to be more skeptical about safety.

Therefore, the study conclusion is that the application of blockchain technology in the medical industry has been recognized and accepted by medical service recipients to some extent. However, there are still problems of information storage security and privacy that need to be improved.

\section{DISCUSSION}

Based on the above questionnaire and experiment, this research project shows that some Chinese consumers are not yet aware of blockchain, and therefore some oppose or abstain from using it. However, they have more or less a general understanding of the application of this technology in the healthcare industry due to the influence of the current COVID-19. Most of the consumers who are aware of blockchain and have some knowledge about it prefer to use it. Also, that they will support it and its application in their lives in the future as well. First of all, they are against or do not give their opinion to people who do not know about blockchain. No one will immediately believe what they don't know and give them their data and private information. But instead, will consumers who understand blockchain and benefit from it deny something that will bring them convenience and benefits because they benefit from it? Most consumers don't understand the benefits of blockchain, such as privacy, security, tamper-proof, convenience, accuracy, data distribution, and storage, so this paper needs to explain and promote it so they understand it. This study considered both consumers and producers would benefit from this. The pharmaceutical industry is one of the many industries that will offer more protection for their private information when consumers embrace blockchain. Not only is it secure, and their data is not easily tampered with, but it will also make it easier for them to seek medical care. Because hospitals can quickly and accurately find their information and prescribe the correct medication when their information is in the blockchain [10]. In summary, although the application of blockchain technology in the medical industry has been recognized and accepted by the recipients of medical services, there is still room for improvement in information storage security and privacy issues.

\section{CONCLUSION}

Through a questionnaire survey on the application of blockchain technology in the medical industry, this study reveals the attitude of medical industry receivers towards the application of blockchain in the medical industry. The questionnaire survey results show that most of the respondents have a certain basic 
understanding of blockchain technology and maintain a supportive attitude towards this application due to the convenience brought by blockchain technology. At the same time, the security of information and the protection of privacy has also aroused widespread concerns.

The analysis of the results of this questionnaire clarifies the degree of recognition and acceptance of the application of blockchain technology in the medical industry. It reveals various problems existing in the current application. The research topic of this paper is the application of blockchain technology in the medical industry, so the results of this paper analysis are closely related to the topic. The analysis results of this paper can provide a reference for decision-makers of institutions or departments in the medical industry to carry out reforms related to the application of blockchain, especially the improvement of privacy protection and information security.

The overall situation of the current COVID-19 has put forward higher and more urgent requirements for the development of blockchain technology in the medical industry. In the process of technological innovation, the recipient of medical services, as an important part of this technology, their opinions and suggestions are important and cannot be ignored. This research topic is just focusing on this point, integrating and analyzing their understanding of this technology, support level, and some suggestions, which can provide a very important reference for the innovation of blockchain technology in the medical industry.

\section{REFERENCES}

[1] Mougayar, William. The business blockchain: promise, practice, and application of the next Internet technology. John Wiley \& Sons, 2016.

[2] Lindman, Juho, Virpi Kristiina Tuunainen, and Matti
Rossi. "Opportunities and risks of Blockchain Technologies-a research agenda." (2017).

[3] Chen Bin, Xu Fang, Chen Hongguang, Wang Shu, Wang Jing. The effect of vulnerability analysis on the transmission of Coronavirus in non-closed hematologic wards [J]. Nursing Research,2020,34(03):371-373.

[4] National Health Commission, PRC. Notice on the issuance of the COVID-19 Diagnostic Protocol (Trial Sixth Edition

[5] 6 Key Blockchain Features You Need to Know Now. 101 Blockchains, 2021.

[6] He, bo, \&amp; Wang, guisheng. Analysis of informatizationed application of medical management based on block chain technology, 2018.

[7] Haq, Ijazul, and Olivier Muselemu Esuka. "Blockchain technology in pharmaceutical industry to prevent counterfeit drugs." International Journal of Computer Applications 180.25 (2018): 8-12.

[8] Han, Xuan, Yong Yuan, and Fei-Yue Wang. "Security problems on blockchain: the state of the art and future trends." Acta Automatica Sinica 45.1 (2019): 206-225.

[9] Mettler, Matthias. "Blockchain technology in healthcare: The revolution starts here." 2016 IEEE 18th international conference on e-health networking, applications and services (Healthcom). IEEE, 2016.

[10] McGhin, Thomas, et al. "Blockchain in healthcare applications: Research challenges and opportunities." Journal of Network and Computer Applications 135 (2019): 62-75. 